IMECE2005-80229

\title{
OPTIMIZED EXCITATION SIGNAL SOURCE FOR EFFICIENT MEASUREMENT OF E/M IMPEDANCE
}

\author{
Buli Xu, PhD Candidate \\ Department of Mechanical Engineering \\ University of South Carolina, Columbia, SC 29208 \\ 803.777.1535,xub@engr.sc.edu
}

\author{
Victor Giurgiutiu, PhD, PE, Member ASME \\ Department of Mechanical Engineering \\ University of South Carolina, Columbia, SC 29208 \\ 803.777.8018, victorg@sc.edu
}

\begin{abstract}
This paper presented an improved algorithm using digitally synthesized excitation signal sources for efficient and accurate measurement of the $\mathrm{E} / \mathrm{M}$ impedance for structural health monitoring with PWAS (piezoelectric wafer active sensor) transducers. Instead of using a sinusoidal excitation to measure the structural $\mathrm{E} / \mathrm{M}$ impedance at a single frequency at a time, the digitally synthesized signal sources get the entire structural E/M impedance spectrum immediately at one step through permanently attached PWAS. Digitally synthesized signal source is a time efficient way for $\mathrm{E} / \mathrm{M}$ impedance spectrum measurement.

Firstly, introduction of E/M impedance measurement and the concept of using transfer function to achieve admittance and impedance of PWAS were provided. Secondly, two ways of synthesizing signal sources were discussed. Thirdly, the characteristics and performance of the two signal sources for $\mathrm{E} / \mathrm{M}$ impedance measurement were compared in simulation and examined by laboratory experiment of measuring a free PWAS impedance spectrum over a wide frequency range $(100 \mathrm{k} \sim 1 \mathrm{MHz})$. Finally, the hardware implementation and measurement precision of the new impedance methods were discussed and concluded.
\end{abstract}

\section{INTRODUCTION}

The electromechanical (E/M) impedance method is a new embedded ultrasonics method that is emerging as an effective and powerful technique for structural health monitoring ${ }^{[2]}$. Using PWAS (piezoelectric wafer active sensor) permanently attached to the structure, the E/M impedance method directly measures the high-frequency local impedance spectrum of the structure. Since high-frequency local impedance spectrum is much more sensitive to incipient damage than low-frequency global impedance, the $\mathrm{E} / \mathrm{M}$ impedance method is considered to be better suited for applications in structural health monitoring than other conventional methods ${ }^{[2]}$.

Although PWAS are small, unobtrusive, and inexpensive, the present impedance measurement equipment such as HP4194 impedance analyzer used in the proof-of-concept demonstration of E/M impedance method is bulky, heavy, and relatively expensive. Several investigators have explored means of reducing the size of the impedance analyzer to make it more compact, even field-portable ${ }^{[3][4][5][7]}$. A portable, chipsize impedance equipment has been proposed for replacing the laboratory HP4199A impedance analyzer ${ }^{[7]}$. HP4194A impedance analyzer measures DUT (Device Under Test) impedance spectrum by exciting pure tones of sinewaves of increasing frequency step by step. Both the DUT's excitation signal and response signal will then be separated into in-phase part and quadrature part using sine correlation algorithm, which is traditionally implemented in analog circuit, for later complex impedance calculation. This method requires recording exact integer numbers cycle of sinewaves at each frequency step which is fairly time consuming. Hence, we proposed and researched on the method using synthesized signal sources for accurately and efficiently measuring E/M impedance. Two different synthetic signal sources will be discussed and compared through both simulation and laboratory experiments. 


\section{THE CONCEPT}

Transfer function, or frequency response, is a mathematical statement that describes the relationship between the input and the output of a system or equipment in terms of the transfer characteristics.

$$
H(f)=\frac{\text { Output }}{\text { Input }}
$$

Using the concept of transfer function, the impedance or admittance of the DUT can be calculated by transforming the time domain excitation signal (voltage $v(t)$ ) and response signal of the DUT (current $i(t))$ to yield the frequency domain quantities $[V(j \omega)$ and $I(j \omega)]$ (Figure 1) using Fourier transform as:

$$
\begin{aligned}
& Y(j \omega)=\frac{I(j \omega)}{V(j \omega)} \\
& Z(j \omega)=\frac{V(j \omega)}{I(j \omega)}
\end{aligned}
$$

Where,

$$
\begin{aligned}
I(j \omega) & =F F T\{i(t)\} \\
V(j \omega) & =F F T\{v(t)\}
\end{aligned}
$$

FFT $\{\cdot\}$ designates the fast Fourier transform.

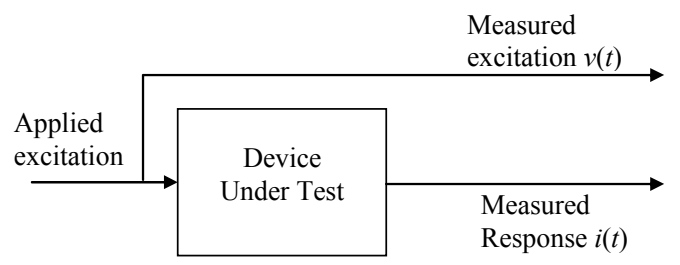

Figure 1 Configuration for impedance measurement using transfer function of DUT ${ }^{[9]}$

Therefore, the impedance spectrum of a DUT can be acquired within one wideband excitation signal sweeping.

\section{SINGAL SOURCE FOR E/M IMEPDANCE MEASUREMENT}

In order to measure the DUT impedance spectrum within one wideband frequency sweeping, proper excitation signals needs to be found out. Two signal sources, the linear chirp signal and the frequency swept signal, have been explored and compared in our research.

\section{LINEAR CHIRP SIGNAL}

Linear chirp signal is a signal whose frequency changes with time linearly. It can be synthesized easily in time domain ${ }^{[11][12]}$. Figure 2a shows a linear chirp with frequency ranging from 0 to $1 \mathrm{MHz}$ recorded in the time domain.

For a sinusoidal signal with a constant frequency $\omega_{0}$, it can be written as:

$$
x(t)=\operatorname{Re}\left\{A e^{j\left(\omega_{0} t+\phi\right)}\right\}=A \cos \left(\omega_{0} t+\varphi_{0}\right)
$$

where $\varphi_{0}$ is the initial phase. The phase is defined as:

$$
\varphi(t)=\omega_{0} t+\varphi_{0}
$$

It can be seen that the phase is a linear function of time and changes at a constant rate that is $\omega_{0}$

$$
d \varphi(t) / d t=\omega_{0}
$$

A general signal can therefore be defined as:

$$
x(t)=\operatorname{Re}\left\{A e^{j \varphi(t)}\right\}
$$

Particularly, a linear chirp signal is produced when the phase $\varphi(t)$ evolves in the format of:

$$
\varphi(t)=\pi \beta t^{2}+2 \pi f_{0} t+\varphi_{0}
$$

where $f_{0}$ is starting frequency, $\varphi_{0}$ is the initial phase, and $\beta$ is a chirp characteristic parameter.

Computing the instantaneous frequency for the chirp, we have

$$
f_{i}(t)=\beta t+f_{0}
$$

Equation (7) shows the instantaneous frequency $f_{i}(t)$ is a linear function of time. The parameter $\beta$ is the rate of frequency change $\left(\beta=\left(f_{1}-f_{0}\right) / t_{1}, f_{1}\right.$ is the frequency corresponding to the time instant $t_{1}$ ) which is used to ensure the desired frequency breakpoint $f_{1}$ at $t_{1}$ can be maintained.

Figure $2 \mathrm{~b}$ is the spectrum of the linear chirp signal. It shows the chirp signal has a continuous quasi-constant frequency-amplitude spectrum within 0 to $1 \mathrm{MHz}$. However, there are unwanted ripples throughout the entire frequency range. Also, the spectrum in certain frequency regions, such as the area closing to $0 \mathrm{~Hz}$ and the area closing to $1 \mathrm{MHz}$, does not maintain a constant.

(a)

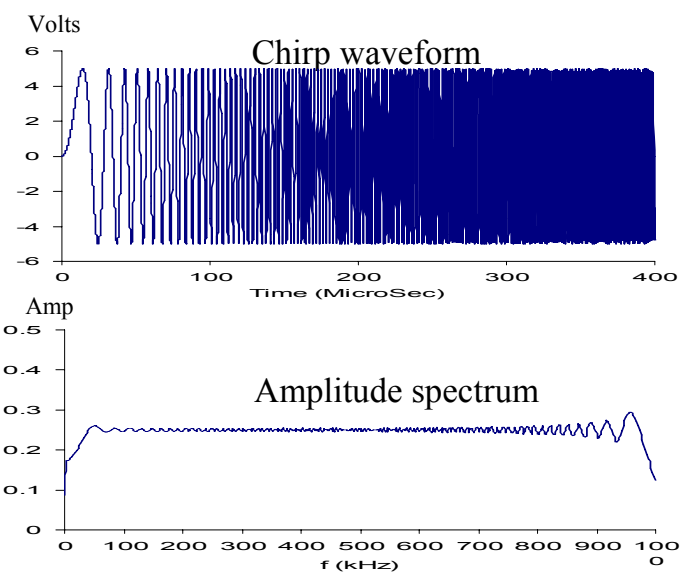

Figure 2 Linear chirp signal (sweep from DC to $1 \mathrm{MHz}$ ): (a) chirp signal in time domain; (b) amplitude spectrum of chirp signal

\section{FREQUENCY SWEPT SIGNAL}

Fourier transform techniques using sweeps as excitation signals are the most advantageous choice for efficient impedance measurement. It measures the response at all 
frequencies within the span simultaneously, thus the source must contain energy at all of the measured frequencies. Therefore, constructing a sweep signal in the spectral domain may avoid the problems of using a linear chirp signal. The synthesis can be implemented by summarizing a series of sinusoidal waves with various amplitudes and phases ${ }^{[12][13]}$ :

$$
v\left(t_{i}\right)=\sum_{k=f_{\text {saut }}}^{f_{\text {end }}} \cos \left(2 \pi k t_{i}+\theta_{k}\right)
$$

Where,

$$
\begin{gathered}
\theta_{k}=\theta_{k-1}+\left(k-f_{\text {start }}\right) \Delta \theta \\
\Delta \theta=-2 \pi /\left(f_{\text {end }}-f_{\text {start }}\right) \\
\theta_{\text {fstart }-1}=0
\end{gathered}
$$

Figure 3a shows a synthesized frequency swept signal sweeping from 0 to $1 \mathrm{MHz}$. Its spectrum in Figure $3 \mathrm{~b}$ manifests the synthesized sweep signal has a perfectly flat amplitude-frequency spectrum over the entire frequency range.

(a)

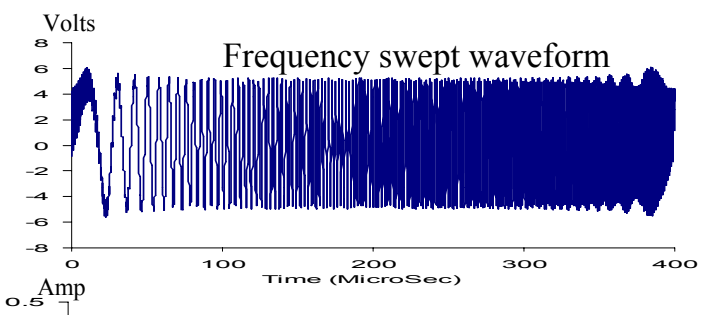

(b)

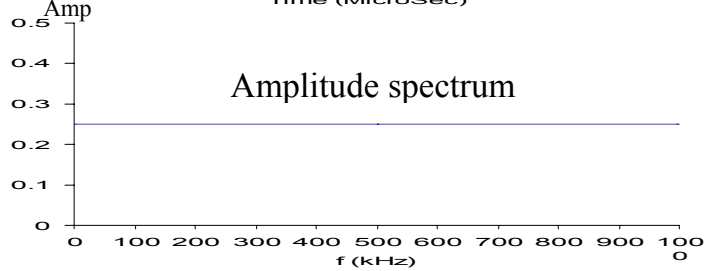

Figure 3 Frequency swept signal: (a) frequency swept signal in time domain; (b) amplitude spectrum of frequency swept signal

\section{SIMULATION AND COMPARISON}

To compare using these two signal sources for $\mathrm{E} / \mathrm{M}$ impedance measurement, a simulation for measuring the impedance spectrum of a free piezoelectric wafer sensor (PWAS) has been conducted. The simulation circuit is shown in Figure 4. A low value resistor $R_{c}$ in series with the free PWAS was employed for current measurement.

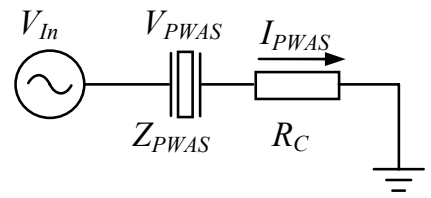

Figure 4 Impedance measurement circuit

According to the Ohm theorem, the voltage across the PWAS, $V_{P W A S}(f)$, and the current flow through the
PWAS, $I_{P W A S}(f)$, can be determined in frequency domain using the following equations respectively:

$$
\begin{gathered}
V_{P W A S}(f)=\frac{Z_{P W A S}(f)}{Z_{P W A S}(f)+R_{c}} V_{I n}(f) \\
I_{P W A S}(f)=\frac{V_{I n}(f)}{Z_{P W A S}(f)+R_{c}}
\end{gathered}
$$

where, $Z_{P W A S}(f)$ designates PWAS impedance.

\section{FREE PWAS IMPEDANCE AND ADMITTANCE MODEL}

For simplicity, 1-D PWAS model was used in simulation $^{[14]}$ :

$$
\begin{aligned}
Z_{P W A S} & =\frac{1}{i \omega \cdot \bar{C}}\left[1-\bar{k}_{31}^{2}\left(1-\frac{1}{\bar{\varphi} \cot \bar{\varphi}}\right)\right]^{-1} \\
Y_{P W A S} & =i \omega \cdot \bar{C}\left[1-\bar{k}_{31}^{2}\left(1-\frac{1}{\bar{\varphi} \cot \bar{\varphi}}\right)\right]
\end{aligned}
$$

where $Y_{P W A S}$ is PWAS admittance, $Z_{P W A S}$ is PWAS impedance, $\bar{k}_{13}^{2}$ is the complex coupling factor; $\bar{C}$ is the capacitance of PWAS; $\bar{\varphi}$ is a notation equal $\frac{1}{2} \gamma l, \gamma$ is the wavenumber, and $l$ is the PWAS length.

(a)

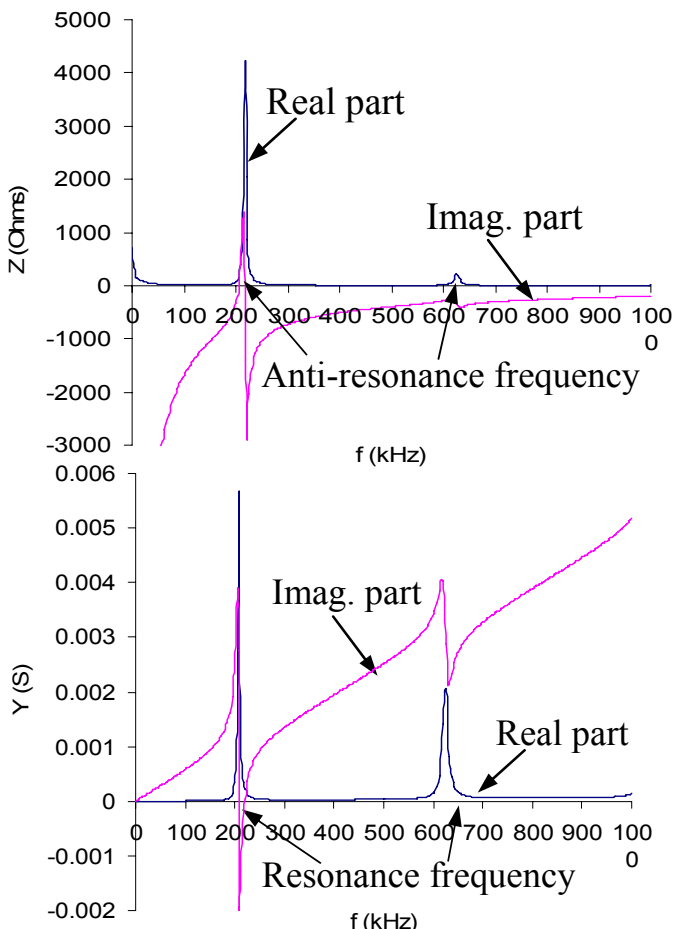

(b)

Figure 5 Numerical simulation of admittance $Y$ and impedance $Z$ responses for 1-D PWAS model: (a) impedance response; (b) admittance response

Figure 5 presents the numerical simulation results of impedance $Z_{P W A S}$ and admittance $Y_{P W A S}$ plotted in real part and imaginary part separately using a free PWAS (APC- 850 piezoceramic) with the dimension of $l=7 \mathrm{~mm}$, $b=1.68 \mathrm{~mm}, t=0.2 \mathrm{~mm}$. Light damping $(\eta=\delta=1 \%)$ was assumed. As shown in the figure, the impedance and 
admittance essentially behave like $Z=1 / i \omega \bar{C}$ or $Y=i \omega \bar{C}$ when outside resonances or anti-resonances. While at resonances or anti-resonances, these basic patterns are modified by adding a pattern of behavior specific to resonance and anti-resonance.

\section{VOLTAGE AND CURRENT SIGANLS IN SIMULATION}

Equation (12) and (13) permit the calculation of amplitude spectrum of voltage, $V_{P W A S}$, and current, $I_{P W A S}$, given the impedance of the PWAS $\left(Z_{P W A S}\right)$. The results are shown in Figure 6.

(a)
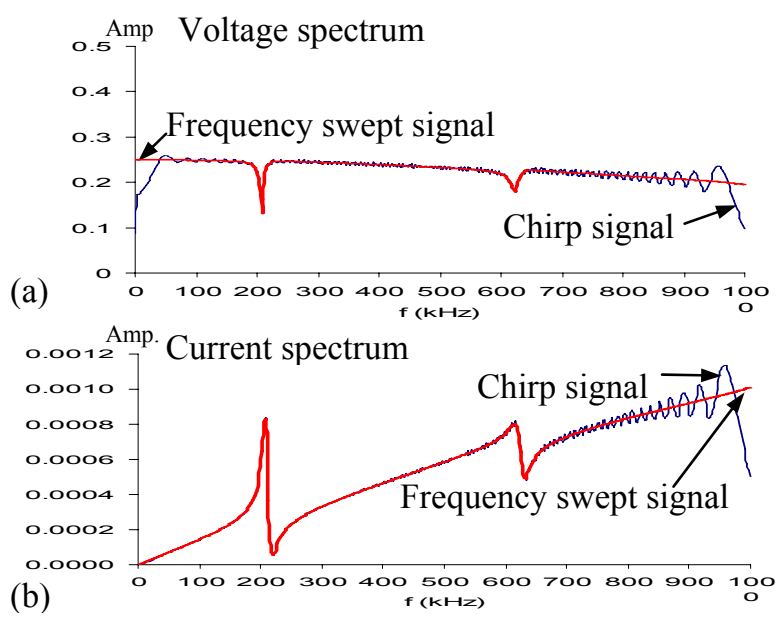

Figure 6 Amplitude spectrum of $V_{P W A S}$ and $I_{P W A S}$ of chirp signal source and frequency swept signal source for free PWAS impedance measurement $\left(f_{s}=10 \mathrm{MHz}, N_{\text {buffer }}=4000\right.$, signal source amplitude equals $5 \mathrm{Vpp}, R_{c}=100 \Omega$ ): (a) Amplitude spectrum of $V_{P W A S}$; (b) Amplitude spectrum of $I_{P W A S}$

As we can see in Figure 6, both the voltage and current spectra of the chirp signal contain ripples, while those of the frequency swept signal are more smooth and have a better shape. Due to the change of PWAS impedance at anti-resonance frequency points and the change of PWAS admittance at resonance frequency points as well, the first valley in voltage spectrum (Figure 6a) was observed at the first resonance frequency point, while the first valley in current spectrum (Figure 6b) was observed at the first anti-resonance frequency point.

Having the voltage spectrum $V_{P W A S}(f)$ and current spectrum $I_{P W A S}(f)$ in the frequency domain, the voltage $V_{P W A S}(t)$ and current $I_{P W A S}(t)$ in time domain can be achieved using inverse Fourier transform. Figure 7 and Figure 8 show the time domain waveforms of $V_{P W A S}(t)$ and $I_{P W A S}(t)$ when using chirp signal source and frequency swept signal source as excitation for the free PWAS impedance measurement respectively. Figure $7 \mathrm{a}$ and Figure $8 \mathrm{a}$ are the excitation voltages for each signal source. A comparison of responding current waveforms in Figure $7 b$ and Figure $8 b$ indicates that frequency swept signal source outputs a large current response than the one output by chirp signal source in low frequency range for impedance measurement. This means the frequency swept signal source should have a higher SNR in low frequency range for impedance measurement.

(a)

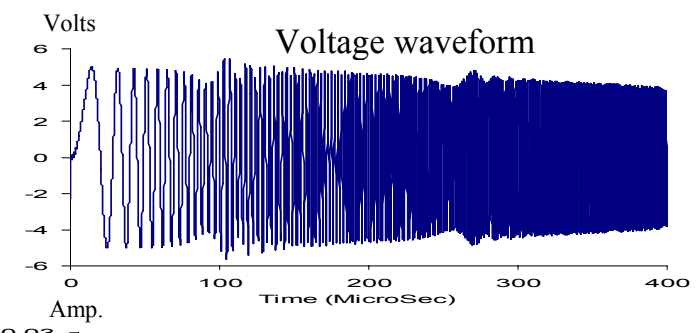

(b)

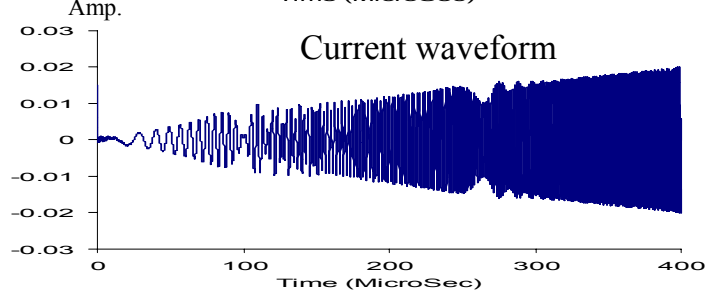

Figure $7 V_{P W A S}(t)$ and $I_{P W A S}(t)$ of chirp signal source for free PWAS impedance measurement:(a) $V_{P W A S}(t) ; \quad$ (b) $I_{P W A S}(t)$

(a)
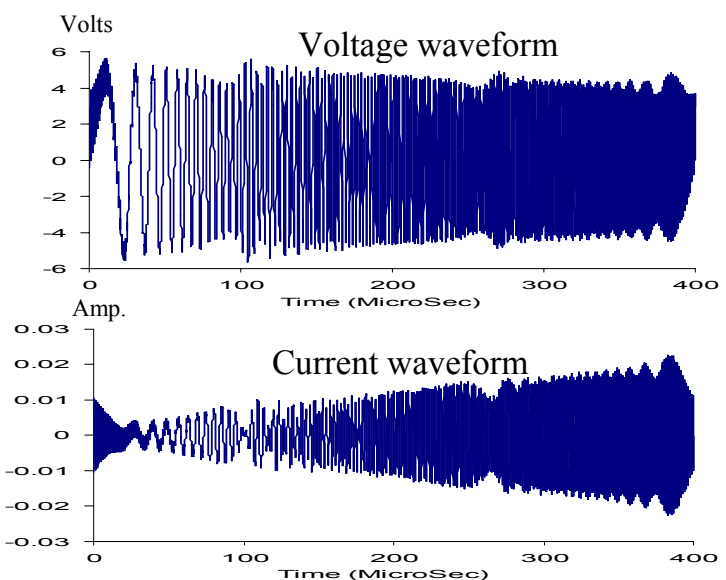

Figure $8 V_{P W A S}(t)$ and $I_{P W A S}(t)$ of frequency swept signal source for free impedance measurement: (a) $V_{P W A S}(t)$; (b) $I_{P W A S}(t)$

\section{LABORATORY EXPERIMENT}

\section{FREE PWAS IMPEDANCE MEASUREMENT}

The practical implementation of impedance measurement system was performed using the hardware configuration in Figure 9. Digitally synthesized signal sources were first uploaded to non-volatile memory slots in the function generator (HP33120A, 12-bit $80 \mathrm{MHz}$ internal D/A converter). The function generator, which was controlled by a LabVIEW program through GPIB card, output uploaded excitation with its frequency equal to the frequency resolution of the digitally synthesized signal source (sample rate/buffer size). The actual 
excitation and the response of the PWAS were recorded synchronously by a two-channel DAQ card (8-bit, $10 \mathrm{MHz}$ sample rate, 4000 points of buffer size) with a certain amount of delay after running of the function generator to ensure the response to stabilize.

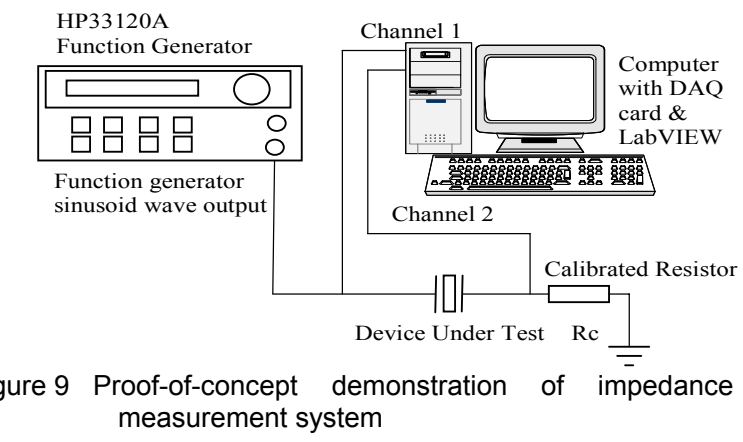

Since the experimental signals are measured in the time domain, the impedance spectrum of the PWAS equals to the Fourier transform of the excitation over the Fourier transform of the response signal according to the equation (3). To improve accuracy and repeatability of measurement, averaging was performed on calculated measurement spectra rather than on the measurements.

Figure 10 shows the spectrum results obtained by using the HP4194A laboratory impedance analyzer and by using the chirp signal as signal source with 256 times of averaging in the frequency domain for measuring the impedance of a free PWAS $(7 \mathrm{~mm}$ diameter, $0.2 \mathrm{~mm}$ thickness). Figure $10 \mathrm{a}$ is the amplitude spectrum of the excitation voltage across the free PWAS and Figure $10 \mathrm{~b}$ is the amplitude spectrum of the measured current response. The real part and imaginary part of the free PWAS impedance spectrums are shown in Figure 10c and Figure 10d using the two methods.

Figure 11 shows the spectrum results obtained by using the HP4194A laboratory impedance analyzer and by using the frequency swept signal as signal source with 256 times of averaging for measuring a free PWAS ( $7 \mathrm{~mm}$ diameter, $0.2 \mathrm{~mm}$ thickness). Figure $11 \mathrm{a}$ and Figure $11 \mathrm{~b}$ are the spectrums of input voltage and output current respectively. The measured spectrum is plotted in real part and imaginary part separately in Figure 11c and Figure 11d separately.

Comparing the spectrum of using the synthesized signal sources in Figure 10c\&d and Figure 11c\&d, it can be seen that both of the synthesized signal sources can acquire the impedance spectrum of a free PWAS very well, even the small peaks. However, for the chirp signal source, small ripples were observed in both voltage and current spectrums in high frequency range (Figure 10a \&b).

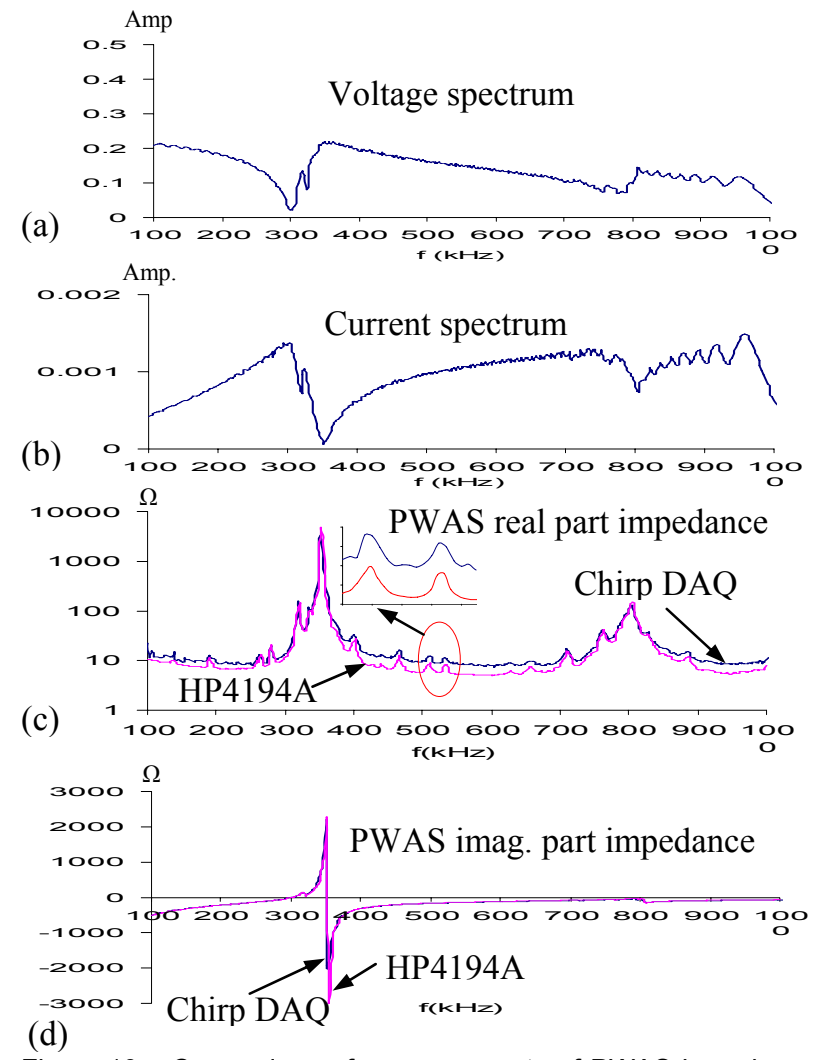

Figure 10 Comparison of measurements of PWAS impedance using HP4194 impedance analyzer and using chirp as the signal source: (a) Amplitude spectrum of voltage across the PWAS; (b) Amplitude spectrum of current; (c) superposed PWAS real part impedance spectrum; (d) superposed PWAS imaginary part impedance spectrum

Also, comparison of the circled parts in the impedance spectra (Figure 10c and Figure 11c) shows that frequency swept signal source had a more smooth impedance spectrum than the one measured by chirp signal source. This indicates that the frequency swept signal may be the better signal source for impedance spectrum measurement than the chirp signal.

Though all precautions have been taken to guarantee high-precision measurement, it still can be noticed that unexplainable small differences exist between the impedance spectra measured by HP4194A impedance analyzer and the new methods using synthetic signal sources (Figure 10c\&d and Figure 11c\&d). The reasons for these differences are not obvious. It is possible that the accuracy of calibrated resistor may have some effects on the difference. Our research work manifests the precision of the new impedance measurement system can be further improved by increasing the buffer size of the system ( so as to increase the spectral resolution) or by decreasing the frequency sweeping range of the synthesized signal sources (i.e., spanning less while sweeping longer in certain frequency range). 


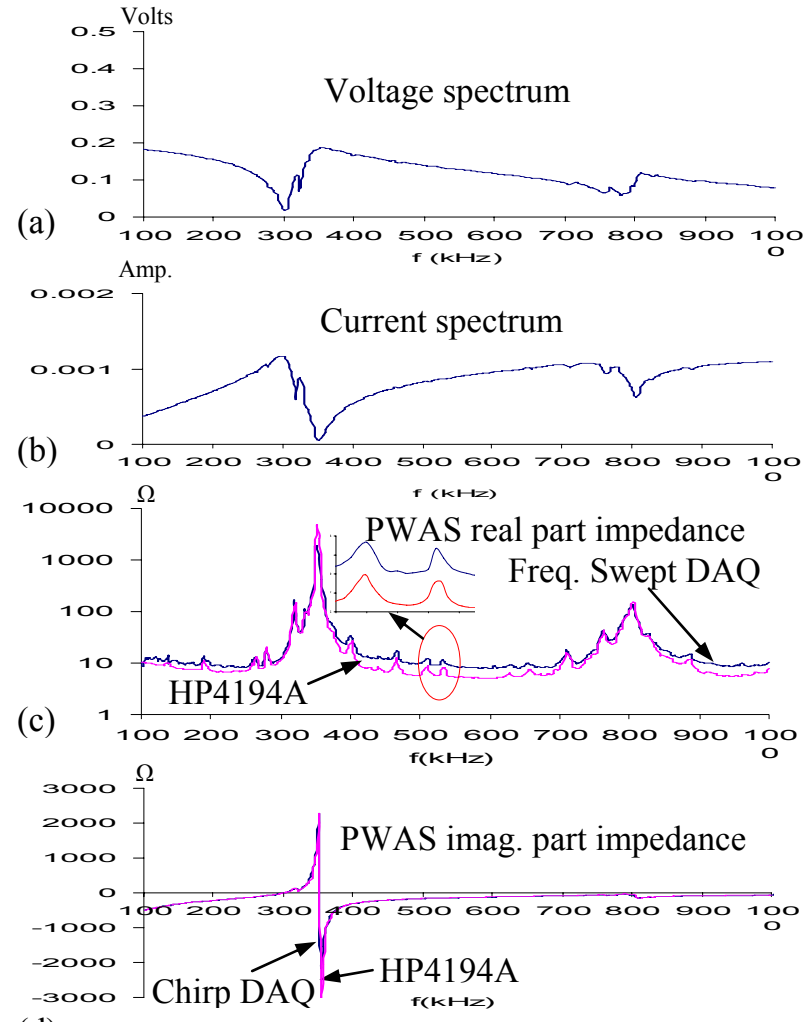

(d)

Figure 11 Comparison of measurements of PWAS impedance by HP4194 impedance analyzer and frequency swept as signal source: (a) Amplitude spectrum of voltage across the PWAS; (b) Amplitude spectrum of current; (c) superposed real part impedance spectrum; (d) superposed imaginary part impedance spectrum

\section{DISCUSSION AND CONCLUSIONS}

Two types of signal sources, the chirp signal and the frequency swept signal, were synthesized and studied in this paper for efficient impedance measurement.

The E/M impedance spectrum was calculated by means of transfer function using Fourier transform since using sweeps as excitation signals are the most advantageous choice for efficient impedance measurement. The synthesized signal source method measures the response at all frequencies within the span simultaneously, thus the source contains energy at all measured frequencies.

As can be seen in simulation, in the time record, the frequency components in the source add up and the peak source amplitude within the time record exceeds the amplitude of each frequency component by about $26 \mathrm{~dB}$ (Figure 3, peak source amplitude 5V, amplitude of each frequency component is $0.25 \mathrm{~V}$ ). Since the input range must be set to accommodate the amplitude peak, each frequency component is measure at $-26 \mathrm{~dB}$ relative to full scale. This is why the spectra of sweep excitation tend to become rather noisy than the one measured by using pure-tone excitations such as HP4194A impedance analyzer. However, this problem can be alleviated by performing averaging of the acquired spectrum over times, by extending the sweep to even longer length to achieve the desired spectral resolution, and by synthesizing an optimized signal source which has a desired power/amplitude spectrum for impedance measurement. Frequency swept signal shows slightly better performance for impedance measurement which has been confirmed both in simulation and experiments. The practical implementation of impedance measurement system was performed by using several pieces of low-cost multipurpose laboratory equipments. It verifies that spectrums obtained by the synthesized signal sources are comparable to the spectrum obtained by the HP4194A analyzer. By using better hardware such as increasing the buffer size and/or by adjusting frequency span range, the precision of using synthetic signal sources can be further improved.

\section{ACKNOWLEDGMENTS}

Financial supports from NASA South Carolina Space Grant Consortium \#520377-3099 and the Air Force Research Lab through UTC Contract \#03-S470-033-C1 of F33615-01-D-5801 are thankfully acknowledged.

\section{REFERENCES}

[1] Park, G., Sohn, H., Farrar, C.R., and Inman, D.J. (2003), "Overview of Piezoelectric Impedance-Based Health Monitoring and Path Forward", The Shock and Vibration Digest, Vol. 35, Issue 6, pp. 451-463, 2003

[2] Giurgiutiu, V.; Zagrai, A. N. (2000a) "Characterization of Piezoelectric Wafer Active Sensors", Journal of Intelligent Material Systems and Structures, Vol. 11, pp. 959-976, 2000

[3] Pardo de Vera, C.; Guemes, J. A. (1997) "Embedded Self-Sensing Piezoelectric for Damage Detection," Proceedings of the International Workshop on Structural Health Monitoring, September 18-20, 1997, Stanford, CA 445-455

[4] Peairs, D.M.; Park, G; Inman, D.J. (2002) "Low Cost Impedance Monitoring Using Smart Materials", Proceeding of the First European Workshop on Structural Health Monitoring, Ecole Normale Superieure, Cachan (Paris), France, July 10-12, 2002

[5] Peairs, D.M.; Park, G; Inman, D.J. (2004) "Improving Accessibility of the impedanceBased Structural Health Monitoring Method", Journal of Intelligent Materials Systems and Structures, Vol. 15, 129-139, Feb. 2004

[6] Grisso1, B. L.; Martin, L. A.; Inman1, D. J. 
(2005), "A Wireless Active Sensing System for Impedance-based Structural Health Monitoring", Proceedings of the IMAC-XXIII:A Conference \& Exposition on Structural Dynamics, January 31 February 3, 2005, Orlando, Florida

[7] Giurgiutiu, V.; Xu, B. (2004) "Development of a Field-Portable Small-Size Impedance Analyzer for Structural Health Monitoring using the Electromechanical Impedance Technique", SPIE's 11th Annual International Symposium on Smart Structures and Materials and 9th Annual International Symposium on NDE for Health Monitoring and Diagnostics, 14-18 March 2004, San Diego, CA. paper \# 5391-923

[8] Macdonald, J. R.(1987), Impedance spectroscopy emphasizing solid materials and systems, Johm Wiley \& Sons, Inc., 1987

[9] NI (1993), Application Note 041, "The Fundamentals of FFT-Based Signal Analysis and Measurement in LabVIEW and LabWindows", NI, Nov., 1993

[10] Stanford Research Systems (2005), "Model SR780 Network Signal AnalyzerOperating Manual and programming Reference", Revision 2.4, 2005

[11] Darowicki, K.; et al (2003), "Continuousfrequency Method of Measurement of Electrode Impedance", Instrumentation Science \& Technology, Vol 31, No. 1, pp 53-62, 2003

[12] Kitayoshi, H.; Sumida, S.; Shirakawa, K.; Takeshita, S. (1985), "DSP Synthesized Signal Source for Analog Testing Stimulus and New Test Method", 1985 International Test conference, P825-834, 1985

[13] Muller, S.; Massarani, P., "TransferFunction Measurement with Sweeps", http://iem.kug.ac.at/ noisternig/iem/bt2004 /ue2/

[14] Giurgiutiu, V.; Lyshevski, S. E. (2004a), Micromechatronics Modeling, Analysis, and Design with MATLAB, CRC Press, 2004 